\title{
Ser 15 of WEE1B is a potential PKA phosphorylation target in G2/M transition in one-cell stage mouse embryos
}

\author{
CHAO LIU ${ }^{1,2}$, YANCHUN LIU ${ }^{1}$, YANG LIU ${ }^{2}$, DIDI WU ${ }^{1}$, ZHIDONG LUAN ${ }^{2}$, \\ ENHUA WANG ${ }^{3}$ and BINGZHI YU ${ }^{1,3}$ \\ ${ }^{1}$ Department of Biochemistry and Molecular Biology, China Medical University, Shenyang; \\ ${ }^{2}$ Department of Developmental Biology, Liaoning Medical University, Jinzhou; \\ ${ }^{3}$ Institute of Pathology and Pathopysiology, China Medical University, Shenyang, Liaoning, P.R. China
}

Received December 17, 2012; Accepted March 28, 2013

DOI: $10.3892 / \mathrm{mmr} .2013 .1437$

\begin{abstract}
The WEE1 kinase family has been shown to be the major kinase family responsible for phosphorylating Tyr 15 on cyclin-dependent kinase 1 (CDK1). WEE1 homolog 2 (WEE2, also known as WEE1B) was first identified in Xenopus laevis and more recently in humans and mice, and is responsible for phosphorylating the CDK1 inhibitory site and maintaining meiotic arrest in oocytes. However, the mechanism by which WEE1B is regulated in one-cell stage mouse embryos remains to be elucidated. In the present study, we examined the role of WEE1B-Ser 15 in $\mathrm{G}_{2} / \mathrm{M}$ transition of one-cell stage mouse embryos. WEE1B-Ser 15 was phosphorylated during the $G_{1}$ and $S$ phases, whereas Ser 15 was dephosphorylated during $G_{2}$ and $\mathrm{M}$ phases in vivo. Overexpression of the phosphor-mimic Wee1B-S15D mutant delayed the re-entry of embryos into mitosis more efficiently than Wee1B-wild type (Wee1B-WT) by direct phosphorylation of CDK1-Tyr 15 . The results of the present study suggested that WEE1B acts as a direct downstream substrate of protein kinase A (PKA) and that Ser 15 of WEE1B is a potential PKA phosphorylation target in the $G_{2} / M$ transition of mouse embryos. In addition, WEE1B inhibits mitosis by negatively regulating $\mathrm{M}$ phase promoting factor activity in one-cell stage mouse embryos.
\end{abstract}

Correspondence to: Professor Bingzhi Yu, Department of Biochemistry and Molecular Biology, China Medical University, 92 Bei Er Road, Heping District, Shenyang, Liaoning 110001, P.R. China

E-mail: ybzbiochem@yeah.net

Abbreviations: CDK1, cyclin-dependent kinase 1; Wee1B-WT, Wee1B-wild type; Wee1B-KD, Wee1B-kinase dead; PKA, protein kinase A; MPF, M phase-promoting factor; PMSG, pregnant mare serum gonadotropin; hCG, human chorionic gonadotropin

Key words: WEE1B, mouse embryo, protein kinase A, M phasepromoting factor, cyclin-dependent kinase 1

\section{Introduction}

M phase-promoting factor (MPF) is a universal regulator of M phase in the eukaryotic cell cycle $(1,2)$. Its activation induces entry into $M$ phase and its subsequent inactivation is necessary to exit from M phase. The activity of MPF in higher eukaryotic cells is primarily regulated by the formation of a complex between the catalytic cyclin-dependent kinase 1 (CDK1, previously known as $\mathrm{CDC} 2$ ) subunit and the regulatory CCNB1 (previously known as cyclin B) subunit, and further by phosphorylation/dephosphorylation at Thr 14 and Tyr 15 of CDK1 associated with CCNB1 (1-4). Until the end of $\mathrm{G}_{2}$ phase, MPF remains inactive through the inhibitory phosphorylation of these residues by the WEE1 kinase family. WEE1 proteins are dual-specificity kinases that uniquely function to phosphorylate specific Tyr/Thr residues on CDKs. There are three WEE1 family members: WEE1 homolog 1 (WEE1, also designated as somatic WEE1A); WEE1 homolog 2 (WEE2, also known as WEE1B) and PKMYT1 (previously known as MYT1). Various genetic and biochemical studies have indicated that WEE1, which was originally identified in the fission yeast Schizosaccharomyces pombe mutant with a small cell or WEE phenotype, is a Tyr-specific protein kinase conserved in all eukaryotes (5). In eukaryotic cells, WEE1A is a nuclear protein that is capable of phosphorylating Tyr 15 but not Thr 14 of CDK1 and thus inhibiting its kinase activity, thereby preventing entry into mitosis (6-9). PKMYT1, a membrane-associated inhibitory kinase, is present in metazoans and phosphorylates CDK1 efficiently on Thr 14 and Tyr 15 residues $(6,10-15)$ and the human PKMYT1 kinase preferentially phosphorylates CDK1 on Thr 14 in a cyclindependent manner (13). WEE1B, a newly identified member of the WEE1 kinase family, is conserved from yeast to humans and has recently been shown to suppress CDK1 activity by phosphorylating its inhibitory sites (16-21). While WEE1A and PKMYT1 are well documented as important regulators of mitosis in multiple cell types in numerous species (22), the role and regulation of WEE1B in zygotes and early embryo development remain to be elucidated.

Protein kinase A (PKA), a cAMP-dependent Ser/Thr kinase, is composed of two catalytic $(C)$ subunits held in an inactive state in association with a regulatory $(\mathrm{R})$ subunit 
dimer. PKA constitutes one of the most important components of several signal transduction pathways, which are important in cell growth, differentiation, proliferation and cell cycle control. Studies in starfish, Xenopus and mammalian oocytes demonstrate that the maintenance of high levels of cAMP and active PKA are important in meiotic arrest (23-26). Previous studies have suggested that PKA may directly phosphorylate and regulate the activity of CDC25B phosphatase $(25,27-29)$ and WEE1A kinase $(20,30,31)$ to form a bidirectional regulatory loop for MPF activity. Generally, cAMP levels are high in meiosis-arrested oocytes and high levels of cAMP may result in high PKA activity, both of which are essential for the maintenance of meiotic arrest (32-34). In particular, when cAMP levels decrease, PKA is deactivated and unable to phosphorylate CDC25 phosphatase, which is subsequently activated. As a result, activated $\mathrm{CDC} 25$ phosphatase translocates to the nucleus where it dephosphorylates CDK1 and thus activates MPF (35,36). The concurrent deactivation of WEE1A kinase allows the inhibitory phosphates to be removed from CDK1 by CDC25 phosphatase $(12,36,37)$. However, the pathway between high PKA activity and the repression of MPF activity has yet to be fully elucidated. To examine the effects of PKA on WEE1B in mammalian cells, WEE1B has been predicted as a potential PKA substrate in mice using the scansite software (http://scansite.mit.edu/) and two potential PKA phosphorylation sites, including Ser 15 and Thr 170 were predicted. The two sites lie within a consensus sequence for PKA (KKLS), which includes a positively charged $\mathrm{R}$ or $\mathrm{K}$ residue upstream of an $\mathrm{S}$ residue. Acting as a candidate target of PKA, it has been demonstrated that Ser 15 is the major phosphorylation site in vitro and is involved in the regulation of meiotic arrest in mouse oocytes by modification of phosphorylation and dephosphorylation (21). However, the role of Ser 15 in the mitotic cell cycle of one-cell stage mouse embryos remains unknown. In the present study, using a highly specific antibody against phospho-Ser 15 of WEE1B in Western blotting, we demonstrated that WEE1B-Ser 15 was phosphorylated during $\mathrm{G}_{1}$ and $\mathrm{S}$ phases, whereas Ser 15 was dephosphorylated during $G_{2}$ and $M$ phases in vivo. Furthermore, we preliminarily examined the role of Ser 15 of WEE1B as the major PKA phosphorylation site in vivo and the inhibitory effects of the kinase are strengthened when this residue is phosphorylated. Collectively, our results suggest that Ser 15 of WEE1B is a potential PKA phosphorylation target in $\mathrm{G}_{2} / \mathrm{M}$ transition of one-cell stage mouse embryos and WEE1B acts as a direct downstream substrate of PKA in mammals. Thus, WEE1B inhibits mitosis by negatively regulating MPF activity.

\section{Materials and methods}

Animals and reagents. Kunming genealogy-specific pathogen-free mice were purchased from the Department of Laboratory Animals, China Medical University [license: SCXK 2008-0005 (Liaoning, China)]. The female and male mice, which were 4 weeks, $18 \mathrm{~g}$ and 8 weeks, $30 \mathrm{~g}$, respectively, were housed under controlled environmental conditions $\left(19^{\circ} \mathrm{C}\right.$ and $12 \mathrm{~h}$ light per day) and provided with food and water ad libitum. The study was performed in strict accordance with the recommendations outlined in the Guide for the Care and Use of Laboratory Animals released by the National Institutes of Health. The protocol for animal handling and the treatment procedures were approved by the Committee on the Ethics of Animal Experiments of the China Medical University. Rabbit anti-WEE1B (mWEE1B-specific antibodies raised in New Zealand white rabbits against the keyhole limpet hemocyanin-conjugated peptide MADTETDQGLNKK), rabbit anti-phospho-WEE1B-pSer 15 (phospho-WEE1B-pSer 15 antibodies raised in New Zealand white rabbits against the keyhole limpet hemocyanin-conjugated phosphor-peptide TDQGLNKKLpSFSF) and rabbit anti-non-phospho-WEE1BpSer 15 (non-phospho-WEE1B-pSer 15 antibodies raised in New Zealand white rabbits against the keyhole limpet hemocyanin-conjugated non-phospho-peptide TDQGLNKKLSFSF) were purchased from Signalway Antibody Co.,Ltd. (Baltimore, MD, USA). Goat anti-pTyr 15 of CDK1, rabbit anti- $\beta$-actin and H-89 were provided by Santa Cruz Biotechnology, Inc. (Santa Cruz, CA, USA). mMESSAGE mMACHINE T7 Ultra kit was purchased from Ambion (Carlsbad, CA, USA) and $\left[\gamma^{-32} \mathrm{P}\right]$ ATP from Peking Yahui Biotechnology Co., Ltd. (Beijing, China). Unless otherwise specified, all other reagents were purchased from Sigma (St. Louis, MO, USA).

Collection and culture of one-cell stage mouse embryos. Mouse embryos at the one-cell stage were collected and cultured as previously described (38). Female mice were injected with 10 IU of pregnant mare serum gonadotropin (PMSG) and then with $10 \mathrm{IU}$ of human chorionic gonadotropin (hCG) 46-48 h post-PMSG, and caged with Kunming males. One-cell stage embryos were obtained from females 18-20 h post-hCG injection and incubated for $2 \mathrm{~min}$ in M2 medium suspended in $300 \mu \mathrm{g} / \mathrm{ml}$ hyaluronidase to remove cumulus cells, washed extensively using M2 medium and then the embryos were microinjected or fixed. Following microinjection, embryos were cultured to the different cell cycle stages in M16 medium at $37^{\circ} \mathrm{C}$ in a humidified atmosphere of $5 \% \mathrm{CO}_{2}$. Embryos pretreated with dibutyryl cyclic AMP (dbcAMP) or H-89 were incubated and collected at different time points. Mitotic stages $\left(\mathrm{G}_{1}, \mathrm{~S}, \mathrm{G}_{2}\right.$ and $\mathrm{M}$ phases) were defined as previously described $(38,39)$ : $\mathrm{G}_{1}$ phase, $12-21 \mathrm{~h}$ post-hCG injection; $\mathrm{S}$ phase, 21-27 h; $\mathrm{G}_{2}$ phase, $27-30 \mathrm{~h}$ and $\mathrm{M}$ phase, 30-33 $\mathrm{h}$. The rate of cleavage, i.e., the number of two-cell embryos resulting from the division of a one-cell embryo, was counted in three independent experiments under a phase contrast microscope at 31,35 or $30 \mathrm{~h}$ post-hCG injection in the absence or presence of dbcAMP and/or H-89.

Plasmid construction and site-directed mutagenesis. The plasmids of wild-type WeelB (pcDNA3.1/V5-His-TOPOWeelB-WT) and kinase-dead WeelB (pcDNA3.1/V5-HisTOPO-WeelB-KD) were kindly provided by Professor Marco Conti (University of California, San Francisco, USA). The pcDNA3.1/V5-His-TOPO-Wee1B-S15A/D construct was prepared by mutating Ser 15 of WeelB to alanine (A) and aspartic acid (D), which acts to mimick phosphorylation. WeelB-WT was used as a template and the site-directed mutagenesis kit (Stratagene, Santa Clara, CA, USA). The primers for WeelBS15A were 5'-CTGAACAAGAAATTAGCCTTCTCCTTT-3' and 5'-GCTAATTTCTTGTTCAGTCCCTGGTCA-3'. The primers used to construct WeelB-S15D were 5'-CTG AACAAGAAATTAGACTTCTCCTTT-3' and 5'-TCTAATTT 
CTTGTTCAGTCCCTGGTCAG-3'. Both recombinant plasmids were sequenced to verify the correct gene insertion and successful mutation.

In vitro transcription. According to our previous study (40), all the pcDNA3.1/V5-His-TOPO constructs were linearized with XhoI as templates for RNA transcription. 5'-capped mRNAs were generated using the mMESSAGE mMACHINE T7 Ultra kit according to the manufacturer's instructions and DNA templates were removed using TURBO DNase. Synthesized mRNAs were dissolved in nuclease-free TE buffer $(5 \mathrm{mM}$ Tris, $0.5 \mathrm{mM}$ EDTA, pH 7.4) and the concentration of mRNAs was determined using a Hitachi U-2900 spectrophotometer (Hitachi High-Technologies Corporation, Tokyo, Japan) or agarose gel electrophoresis.

Synchronizing the embryos and microinjection. To synchronize the embryos, embryos were placed in a drop of M2 medium under mineral oil in the lid of a $3-\mathrm{cm}$ Falcon culture dish and synchronized for $7 \mathrm{~h}$ following microinjection and release from a thymidine block. Alternatively, asynchronous cells were recorded $20 \mathrm{~h}$ following microinjection. The typical injection volumes were $5 \%$ (10 pl, cytoplasmic) of the total cell volume of embryos. Non-microinjection or microinjection with TE buffer were selected as controls. Following microinjection, embryos were transferred to M16 medium and incubated at $37^{\circ} \mathrm{C}$ in $5 \% \mathrm{CO}_{2}$, and the percentage of cell cleavage was scored under a dissecting microscope. Morphological analysis was performed at the indicated times.

Western blotting. Protein was extracted from $\sim 160$ mouse embryos using $20 \mu \mathrm{l}$ protein extraction buffer $(150 \mathrm{mM} \mathrm{NaCl}$, $1 \mathrm{mM} \mathrm{Na}{ }_{2}$ EDTA, $1 \mathrm{mM}$ EGTA, $1 \%$ Triton, $2.5 \mathrm{mM}$ sodium pyrophosphate, $1 \mathrm{mM} \beta$-glycerophosphate, $1 \mathrm{mM} \mathrm{Na} \mathrm{VO}_{4}$, $1 \mu \mathrm{g} / \mathrm{ml}$ leupeptin and $1 \mathrm{mM}$ PMSF). The protein extracts were separated by $10 \%$ polyacrylamide gel electrophoresis and the protein was transferred onto polyvinylidene fluoride membranes. The membranes were blocked using 3\% (w/v) BSA in Tris-buffered saline containing $0.05 \%$ Tween-20 for $1 \mathrm{~h}$ at room temperature and incubated with primary antibody overnight at $4^{\circ} \mathrm{C}$. The blots were then incubated with HRP-linked secondary antibody and visualized using the enhanced chemiluminescence detection system (Pierce Biotechnology, Inc., Rockford, IL, USA).

Assay of MPF activity. MPF kinase activity was assayed as previously described $(41,42)$. Ten embryos were collected in $5 \mu \mathrm{l}$ collection buffer (PBS containing $1 \mathrm{mg} / \mathrm{ml}$ polyvinyl alcohol, $5 \mathrm{mM}$ EDTA, $10 \mathrm{mM} \mathrm{Na}_{3} \mathrm{VO}_{4}$ and $10 \mathrm{mM} \mathrm{NaF}$ ) and immediately stored at $-70^{\circ} \mathrm{C}$ until the kinase assay was performed. Embryos were lysed by three rounds of freezing and thawing in $20 \mu \mathrm{l}$ of extraction buffer $[50 \mathrm{mM}$ Tris- $\mathrm{HCl}$ (pH 7.4), $250 \mathrm{mM} \mathrm{NaCl}, 1 \mathrm{mM}$ EDTA, $50 \mathrm{mM}$ NaF, $1 \mathrm{mM}$ DTT, $0.1 \%$ Triton X-100, $10 \mu \mathrm{M}$ leupeptin, $100 \mu \mathrm{g} / \mathrm{ml}$ aprotinin and $0.5 \mu \mathrm{l}$ PMSF]. Embryo lysate $(5 \mu \mathrm{l})$ was added to the $20 \mu \mathrm{l}$ MPF buffer [250 mM Tris- $\mathrm{HCl}$ (pH 7.5), $50 \mathrm{mM} \mathrm{MgCl} 2,5 \mathrm{mM}$ EGTA, 10 mM DTT, 200 mM $\beta$-glycerophosphate, $100 \mathrm{mM}$ p-nitrophenylphosphate, $0.5 \mathrm{mM}$ sodium vanadate, $0.05 \%$ Brij 35, 2 mg/ml histone (type III-S), $2.4 \mu \mathrm{M}$ PKA inhibitor (PKI), $0.5 \mathrm{mM}$ ATP and $\left.10 \mathrm{mCi} / \mathrm{ml}\left[\gamma_{-}{ }^{32} \mathrm{P}\right] \mathrm{ATP}\right]$, and incu- bated at $30^{\circ} \mathrm{C}$ for $10 \mathrm{~min}$. Then, $25 \mu \mathrm{l}$ of reaction mixture was spotted on Whatman p81 paper and the reaction was stopped using 5\% $\mathrm{H}_{3} \mathrm{PO}_{4}$ solution. Following thorough washing, the radioactivity on the filter paper was counted using a Beckman scintillation counter.

A parallel incubation was performed to confirm the phosphorylation of histone H1. Protein extracted from 20 embryos was incubated with $50 \mu \mathrm{l}$ of MPF buffer containing $20 \mathrm{mCi} / \mathrm{ml}$ $\left[\gamma^{-32} \mathrm{P}\right]$ ATP at $37^{\circ} \mathrm{C}$ for $30 \mathrm{~min}$ and the reaction was stopped by adding an equal amount of $2 \mathrm{X}$ SDS buffer. The mixtures were then separated by a $12 \%$ SDS-PAGE and the incorporation of ${ }^{32} \mathrm{P}$ into histone $\mathrm{H} 1$ was visualized by autoradiography.

Statistical analysis. Experiments were performed independently at least three times. Values were analyzed by one-way analysis of variance or Student's t-test with GraphPad Prism 5 software. $\mathrm{P}<0.05$ was considered to indicate a statistically significant result.

\section{Results}

Phosphorylation status of WEE1B-Ser 15 in one-cell stage mouse embryos. To identify whether WEE1B-Ser 15 was phosphorylated in vivo, we collected one-cell stage mouse embryos at different phases in the absence or presence of dbcAMP and/or H-89, then measured the phosphorylation status of WEE1B-Ser 15 using the phosphor-specific antibody and non-phosphorylation status using the non-phosphorspecific antibody. On the basis of previous experimental studies $(27,40), 2 \mathrm{mmol} / \mathrm{l}$ of dbcAMP, a PKA activator, led to maximal $G_{2}$ arrest, suggesting inhibition of the $G_{2} / M$ transition in one-cell stage mouse embryos. It has been well documented that $\mathrm{H}-89$ inhibits the PKA $\mathrm{C}$ subunit and readily diffuses through the cell membrane $(43,44)$. Previously, we $(27)$ demonstrated that $40 \mu \mathrm{mol} / 1 \mathrm{H}-89$ induced all of the mouse embryos to enter the $\mathrm{M}$ phase of mitosis, suggesting activation of the $\mathrm{G}_{2} / \mathrm{M}$ transition in one-cell stage mouse embryos. The results demonstrated that a phosphorylated WEE1B-Ser 15 band was observed at $G_{1}$ and $S$ phases, whereas no phosphorylation of WEE1B-Ser 15 was observed at $\mathrm{G}_{2}$ and $\mathrm{M}$ phases in one-cell stage embryos with or without H-89 (Fig. 1A and C). The strong phosphorylated WEE1B-Ser 15 band was detected in the $\mathrm{G}_{1}$ and $S$ phases, and also in the $G_{2}$ and $M$ phases in the presence of dbcAMP (Fig. 1B). Conversely, a non-phosphorylated WEE1B-Ser 15 band was mainly detected during the $G_{2}$ and $\mathrm{M}$ phases, and is strongest in the presence of H-89 (Fig. 1C). Therefore, these results demonstrated that WEE1B-Ser 15 is phosphorylated at $\mathrm{G}_{1}$ and $\mathrm{S}$ phases and dephosphorylated at $\mathrm{G}_{2}$ and $\mathrm{M}$ phases in vivo.

WeelB-WT and Wee1B-S15D inhibit MPF activity. To investigate the roles of Ser 15 phosphorylation of WEE1B, we mutated Ser 15 of WEE1B and generated Wee1B-15A and Wee1B-15D mutants. Various WEE1B mutants were overexpressed in one-cell stage mouse embryos. Immunoblotting with anti-WEE1B antibody confirmed that the WEE1B protein accumulated at higher levels in WeelB-WT/KD and WeelB15A/D mRNA-microinjected embryos compared with the control, $5 \mathrm{~h}$ following microinjection (Fig. 2A), demonstrating that the exogenous mRNA of four types of WeelB were able 
A $\mathrm{pSer} 15 \stackrel{\mathrm{G} 1}{=} \mathrm{S} \quad \mathrm{G} 2 \quad \mathrm{M}$

\section{Non-pSer 15}

B pSer 15

Non-pSer 15

C pSer15

Non-pSer 15

Figure 1. The phosphorylated and unphosphorylated status of WEE1B-Ser 15 in one-cell stage mouse embryos at different phases of the cell cycle in the absence/presence of $2 \mathrm{mmol} / 1 \mathrm{dbcAMP}$ and $40 \mu \mathrm{mol} / 1 \mathrm{H}-89$. A total of 160 embryos were loaded onto each lane in Western blotting. Gels were then transferred to the PVDF membrane and probed with phosphor-WEE1B-Ser 15 and non-phosphor-WEE1B-Ser 15 antibodies, respectively. (A) Western blotting analysis of the phosphorylated and non-phosphorylated status of WEE1B-Ser 15 in mouse embryos during $\mathrm{G}_{1}, \mathrm{~S}, \mathrm{G}_{2}$ and $\mathrm{M}$ phases with a phosphor-specific and a non-phosphor-specific WEE1B-Ser 15 antibody, respectively, in the absence of dbcAMP and H-89. (B) The phosphorylated and unphosphorylated status of WEE1B-Ser 15 during $\mathrm{G}_{1}, \mathrm{~S}, \mathrm{G}_{2}$ and $\mathrm{M}$ phases were measured in the presence of $2 \mathrm{mmol} / \mathrm{l} \mathrm{dbcAMP}$. (C) The phosphorylated and unphosphorylated form of WEE1B-Ser 15 at $\mathrm{G}_{1}, \mathrm{~S}, \mathrm{G}_{2}$ and $\mathrm{M}$ phases were detected in the presence of $40 \mu \mathrm{mol} / \mathrm{l} \mathrm{H}-89$. dbcAMP, dibutyryl cyclic AMP.

A

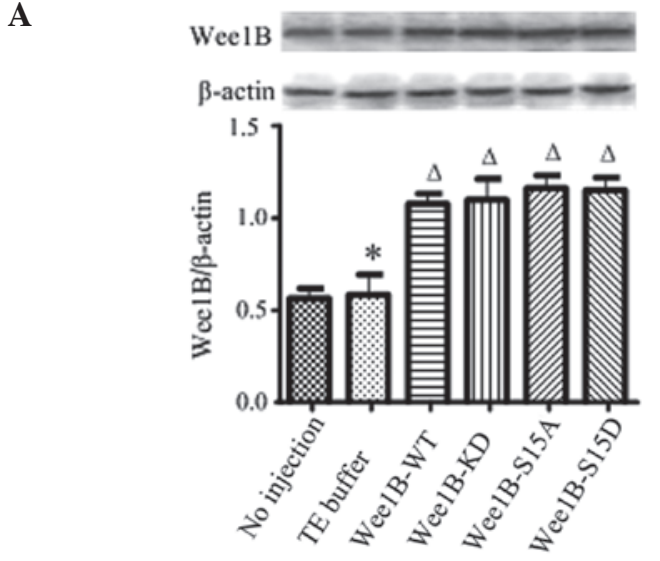

B

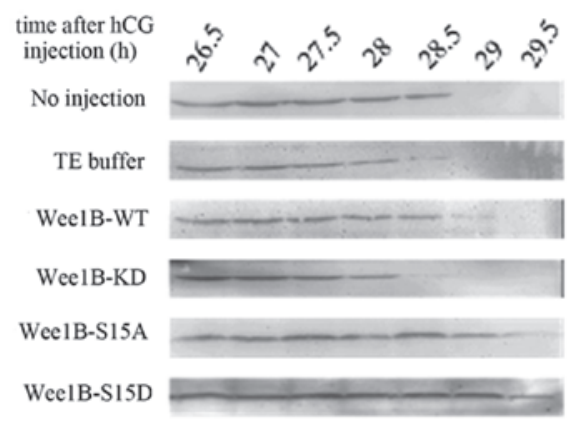

C

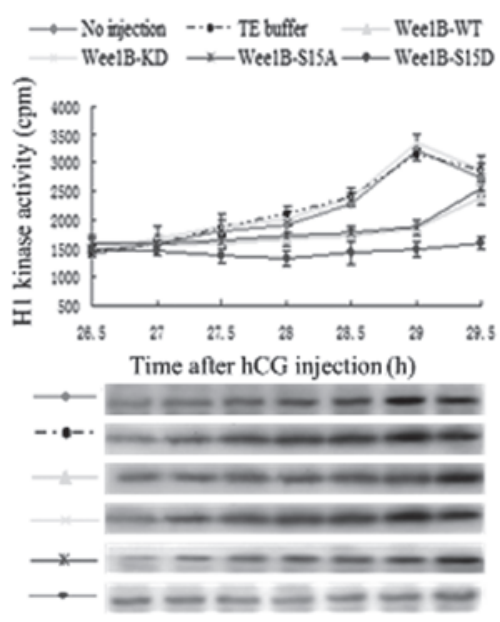

D

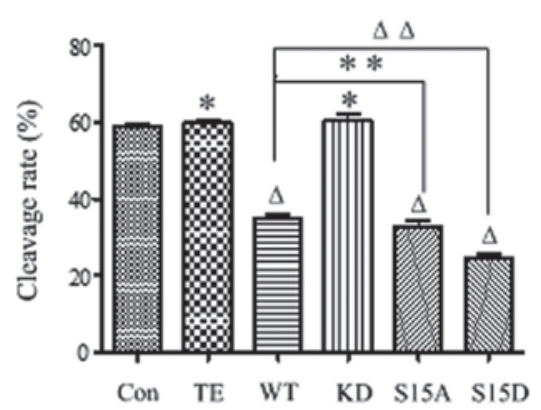

Figure 2. Phosphorylation on Ser 15 is essential for WEE1B. (A) Western blot analysis of WEE1B expression $5 \mathrm{~h}$ following microinjection of Wee1B mRNA (upper panel). A total of $0.03 \mathrm{ng}$ mRNA encoding WeelB-WT/KD and WeelB-S15A/S15D were microinjected into each embryo. Control embryos were microinjected with or without TE buffer. Western blotting was performed using anti-WEE1B antibody in different microinjected groups. $\beta$-actin was used as an internal control. Band intensities for WEE1B were quantified and normalized to $\beta$-actin level (bottom panel). Each value was expressed as mean \pm SEM from three independent experiments. ${ }^{\Delta} \mathrm{P}<0.01$ and ${ }^{*} \mathrm{P}<0.05$, compared with no injection group. (B) Western blot analysis of the phosphorylation status of CDK1-Tyr 15 in the control and various WeelB mRNA-injected embryos. The embryos were collected at 26.5, 27, 27.5, 28, 28.5, 29 and 29.5 h post-hCG injection. A total of 160 embryos were lysed and then subjected to western blot analysis. A representative of three independent experiments is shown. (C) MPF activity in embryos injected with various WeelB mRNAs and controls. Embryos in controls were microinjected with or without TE buffer. The embryos were collected at 26.5, 27, 27.5, 28, 28.5, 29 and $29.5 \mathrm{~h}$ post-hCG injection. For each point, 10 embryos were collected and MPF activity was examined by scintillation counting (upper panel) and autoradiography (bottom panel). Each value is expressed as the mean \pm SEM and shown is a representative of three independent experiments. (D) The cleavage rate in cultured mouse embryos injecting various WeelB mRNAs and controls at $31 \mathrm{~h}$ post-hCG injection. The cleavage rate was calculated and each value was expressed as the mean \pm SEM from three independent experiments. ${ }^{\Delta} \mathrm{P}<0.01$ and ${ }^{*} \mathrm{P}<0.05$, compared with the no injection group. WeelB-WT, WeelB-wild type; WeelB-KD, WeelB-kinase dead; MPF, M phase-promoting factor; hCG, human chorionic gonadotropin; CDK1, cyclin-dependent kinase 1 . 
to be translated efficiently in one-cell stage mouse embryos. Furthermore, there was no difference among the microinjection groups.

To investigate the effects of WeelB on the phosphorylation status of CDK1-Tyr 15 in mouse embryos, western blotting was carried out at 26.5, 27, 27.5, 28, 28.5, 29 and $29.5 \mathrm{~h}$ post-hCG injection in the control and WeelB mRNA-microinjected embryos (Fig. 2B). In the control group, there were strong signals of CDK1-Tyr 15 phosphorylation at 26.5-28 h, a reduced phosphorylation level at $28.5 \mathrm{~h}$ and no signal at $29 \mathrm{~h}$ post-hCG injection, which demonstrated that MPF was fully activated. Moreover, there was a weak CDK1-Tyr 15 phosphorylation signal at $29 \mathrm{~h}$ and no signal at $29.5 \mathrm{~h}$ post-hCG injection in the WeelB-WT and S15A-injected embryos. When Wee $1 B$-KD was overexpressed, strong signals of CDK1-Tyr 15 phosphorylation were detected at $26.5 \mathrm{~h}$; however, no signal was detected at $29 \mathrm{~h}$ post-hCG injection, as in the control. By contrast, the inhibitory phosphorylation signals of CDK1-Tyr 15 were still observed at $29.5 \mathrm{~h}$ post-hCG injection in the WeelB-S15D-overexpressed embryos. These results were consistent with the changes of MPF activity, suggesting that Ser 15 phosphorylation of WEE1B is critical for the regulation of MPF. Furthermore, to identify the correlation between MPF activity and phosphorylation status of CDK1-Tyr 15, we measured the MPF activity at $26.5 \mathrm{~h}$ post-hCG injection every $30 \mathrm{~min}$. In the control group, MPF activity was gradually increased at $28.5 \mathrm{~h}$ post-hCG injection, reaching its maximal level at $29 \mathrm{~h}$ before gradually decreasing. In the WeelB-WT and S15A mRNA-microinjected embryos, MPF activity reached its peak value at $29.5 \mathrm{~h}$ post-hCG injection, $\sim 30$ min later than the control group. In the WeelB-KD mRNA-microinjected embryos, MPF activity reached its peak value at $29 \mathrm{~h}$ post-hCG injection, which was similar to that observed in the control group. However, MPF activity remained consistently low until $29.5 \mathrm{~h}$ post-hCG injection in the Wee1B-S15D mRNA-injected embryos (Fig. 2C). These results were consistent with the changes in the phosphorylation status of CDK1-Tyr 15.

WeelB-WT and Wee1B-S15D decrease the cleavage of one-cell stage embryos. To examine the role of Ser 15 phosphorylation of WEE1B during the cleavage of one-cell stage embryos, the division of mouse embryos was observed at 26.5-29.5 h post-hCG injection, and the cleavage rate was calculated at $31 \mathrm{~h}$. In the control group, cleavage of the embryos started at 28.5-29 h post-hCG injection and 58.97 (no injection) and $59.87 \%$ (TE injection) of embryos had reached the two-cell stage at $31 \mathrm{~h}$. Embryos microinjected with WeelB-WT mRNAs entered M phase until 29-29.5 h post-hCG injection, and the cleavage rates were $35.1 \%$ at $31 \mathrm{~h}$. Embryos microinjected with mRNA of WeelB-KD entered M phase at 28.5-29 h posthCG injection, and almost $60.33 \%$ of embryos had reached the two-cell stage at $31 \mathrm{~h}$, similar to the control group ( $\mathrm{P}>0.05)$. By contrast, embryos injected with WeelB-S15A mRNA had reached the two-cell stage at 29-29.5 h post-hCG injection, and the cleavage rate was $32.78 \%$ at $31 \mathrm{~h}$, markedly lower than that of the control $(\mathrm{P}<0.01)$; however, similar to the WeelB-WT mRNA-injected embryos ( $>>0.05)$. However, embryos microinjected with WeelB-S 15D mRNAs rarely entered M phase at $29.5 \mathrm{~h}$ post-hCG injection, and the cleavage rate was $\sim 24.77 \%$ at $31 \mathrm{~h}$, markedly lower than that of the control group $(\mathrm{P}<0.01$; Fig. 2D). These results demonstrated that the phosphorylation of the Ser 15 residue of WEE1B is essential for its activity during the cleavage of one-cell stage embryos.

Microinjection of WeelB mRNA suppresses MPF activity induced by $d b c A M P$. To further examine whether PKA is capable of phosphorylating Ser 15 of WEE1B, various WeelB mRNAs were microinjected into embryos during $S$ phase in the presence of $2 \mathrm{mmol} / \mathrm{l} \mathrm{dbcAMP}$. The WEE1B protein was highly expressed at $5 \mathrm{~h}$ in each mRNA-microinjected embryo compared with the control (Fig. 3A) and there was no significant difference among the microinjection groups, indicating that various exogenous WeelB mRNAs were able to be translated efficiently in mouse embryos.

The phosphorylation status of CDK1-Tyr 15 was detected by western blotting at 29, 30, 31, 32, 33, 34 and $35 \mathrm{~h}$ post-hCG injection in various exogenous WeelB mRNAs in the presence of $2 \mathrm{mmol} / \mathrm{l} \mathrm{dbcAMP}$. In the control and WeelB-KD mRNAinjected embryos, strong bands of phosphorylated CDK1-Tyr 15 were identified at 29-35 h post-hCG injection, indicating that MPF activity was inhibited. In the WeelB-WT/S15A-injected embryos, weak CDK1-Tyr 15 phosphorylation was detected at $35 \mathrm{~h}$ post-hCG injection, which was coincident with MPF activity. However, when Wee1B-S15D was overexpressed, a strong signal was present at $35 \mathrm{~h}$ post-hCG injection, suggesting that MPF was completely inactivated (Fig. 3B). The results suggested that Ser 15 phosphorylation of WEE1B is required for PKA-induced CDK1-Tyr 15 phosphorylation and therefore for PKA-induced MPF inhibition.

To investigate the effects of Ser 15 phosporylation on MPF inhibition induced by PKA in mouse embryos, MPF activity was measured beginning at $29 \mathrm{~h}$ post-hCG injection at 30-min intervals. As shown in Fig. 3C, the MPF activity was stably low at 29-35 $\mathrm{h}$ in the control and WeelB-KD mRNA-injected embryos, which indicated that MPF activity was inhibited by PKA activation. Additionally, MPF activity in Wee1B-WT/S15A mRNA-injected embryos increased weakly at $35 \mathrm{~h}$ post-hCG injection. By contrast, MPF activity in embryos injected with the WeelB-S15D mutants remained at a low level at 29-35 h post-hCG injection, indicating $\mathrm{G}_{2} / \mathrm{M}$ arrest. These results demonstrated that WEE1B functions downstream of PKA.

Overexpression of WeelB prohibits the mitotic entry of mouse embryos treated by dbcAMP. In the presence of $2 \mathrm{mmol} / \mathrm{l}$ dbcAMP, the cleavage of mouse embryos was observed at 29-35 h post-hCG injection, and the cleavage rate was calculated at $35 \mathrm{~h}$. As shown in Fig. 3D, the cleavage rate of embryos treated with dbcAMP in each group evidently decreased, and extremely few embryos reached the two-cell stage at $35 \mathrm{~h}$ post-hCG injection in the control, WeelB-WT/KD and WeelBS15A-microinjected embryos. No embryos microinjected with WeelB-S15D mRNA entered M phase at $35 \mathrm{~h}$.

WeelB-S15A/D inhibits MPF activity induced by $H-89$. Previously, we and other authors have demonstrated that H-89 activates MPF through the inhibition of PKA $(27,43,44)$. To investigate the role of Ser 15 phosphorylation of WEE1B during PKA-induced MPF inhibition, various WeelB mRNAs 
A

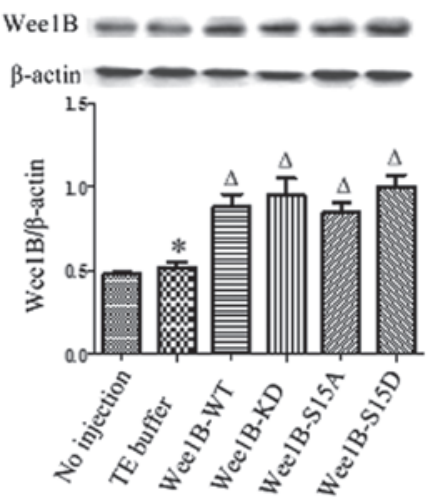

B

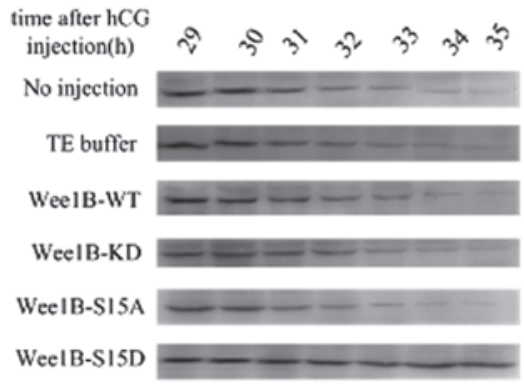

C

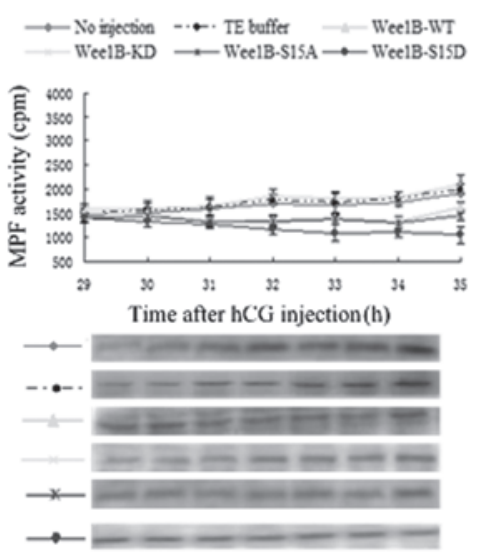

D

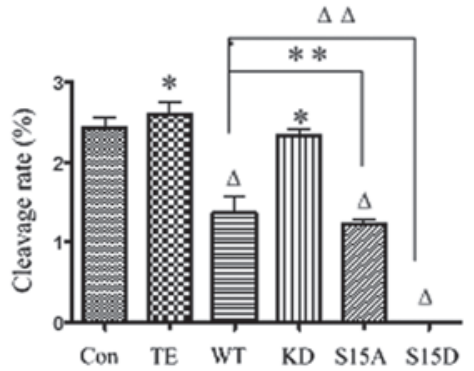

Figure 3. WEE1B functions downstream of PKA. (A) Western blotting analysis of WEE1B expression at $5 \mathrm{~h}$ following microinjection of WeelB mRNA in the presence of $2 \mathrm{mmol} / 1 \mathrm{dbcAMP}$. In total, $0.03 \mathrm{ng}$ of mRNA encoding Wee1B-WT/KD and Wee1B-S15A/S15D were microinjected into each embryo. Embryos in the control group were microinjected with or without TE buffer. Western blotting was performed using anti-WEE1B antibody in different microinjected groups. $\beta$-actin was used as an internal control. Band intensities for WEE1B were quantified and normalized to $\beta$-actin level (bottom panel). Each value is expressed as the mean \pm SEM from three independent experiments. ${ }^{\Delta} \mathrm{P}<0.01$ and ${ }^{*} \mathrm{P}<0.05$, compared with the no injection group. (B) Western blotting analysis of the phosphorylation status of CDK1-Tyr 15 in the control and various WeelB mRNA-injected embryos in the presence of $2 \mathrm{mmol} / \mathrm{ldbcAMP}$. The embryos were collected at 29,30,31,32,33, 34 and $35 \mathrm{~h}$ post-hCG injection. A total of 160 embryos were loaded onto each lane and fractionated on $12 \%$ SDS-PAGE. Gels were then transferred to the PVDF membrane and probed with CDK1-Tyr 15 antibody. Shown is a representative of three independent experiments. (C) MPF activity in embryos injected with various Wee1B mRNAs and controls. Embryos in controls were microinjected with or without TE buffer. Embryos in the presence of $2 \mathrm{mmol} / \mathrm{ldbcAMP}$ were collected at 29, 30,31, 32, 33, 34 and $35 \mathrm{~h}$ post-hCG injection. For each point, 10 embryos were collected and MPF activity was examined by scintillation counting (upper panel) and autoradiography (bottom panel). Each value is expressed as the mean \pm SEM, shown is a representative of three independent experiments. (D) The cleavage rate in cultured mouse embryos injected with various WeelB mRNAs and controls at $35 \mathrm{~h}$ post-hCG injection in the presence of $2 \mathrm{mmol} / \mathrm{l} \mathrm{dbcAMP}$. The cleavage rate was calculated and each value was expressed as mean \pm SEM from three independent experiments. ${ }^{\Delta} \mathrm{P}<0.01$ and ${ }^{*} \mathrm{P}<0.05$, compared with the no injection group. WeelB-WT, WeelB-wild type; WeelB-KD, WeelB-kinase dead; MPF, $\mathrm{M}$ phase-promoting factor; $\mathrm{hCG}$, human chorionic gonadotropin; CDK1, cyclin-dependent kinase 1.

were microinjected into mouse embryos during $\mathrm{S}$ phase in the presence of $40 \mu \mathrm{mol} / 1 \mathrm{H}-89$. The WEE1B protein was more highly expressed at $5 \mathrm{~h}$ in the mRNA-microinjected embryos than in the control group (Fig. 4A). No significant differences among the microinjection groups were observed, indicating that the various exogenous WeelB mRNAs were able to be translated efficiently in mouse embryos.

To identify the phosphorylation of CDK1-Tyr 15 in the mouse embryos treated with H-89, the embryos were collected at 26, 26.5, 27, 27.5, 28, 28.5 and 29 h post-hCG injection and subjected to western blotting. The inhibitory phosphorylation signals of CDK1-Tyr 15 were detected at 26-27 h, and no phosphorylation band was detected at $27.5 \mathrm{~h}$ post-hCG injection in the control and WeelB-KD mRNAinjected embryos (Fig. 4B). However, in the WeelB-WT and Wee1B-S15A mutant-injected embryos, there were strong bands of CDK1-Tyr 15 phosphorylation at $26 \mathrm{~h}$, and weak signals were detected at $28 \mathrm{~h}$ post-hCG injection. Conversely, the inhibitory phosphorylation bands of CDK1-Tyr 15 were observed at 26-28.5 $\mathrm{h}$ when WeelB-S15D was overexpressed, and there was still a strong CDK1-Tyr 15 phosphorylation signal detected at $29 \mathrm{~h}$ post-hCG injection.
In the presence of $\mathrm{H}-89$, MPF was initially detected at $26 \mathrm{~h}$ post-hCG injection at $30-\mathrm{min}$ intervals. The MPF activity oscillated in different groups. In the control, there was a marked increase in MPF activity at $27.5 \mathrm{~h}$ post-hCG injection, then MPF activity peaked at $28 \mathrm{~h}$, indicating that inhibition of PKA activates MPF early. Furthermore, MPF activity in WeelB-KD mRNA-injected embryos reached its maximal level at $28 \mathrm{~h}$ post-hCG injection, then decreased gradually, as in the control. However, MPF activity in embryos injected with WeelB-WT and WeelB-S15A mRNAs peaked at $28.5 \mathrm{~h}$ and the MPF peak lagged behind the control. However, MPF activity in embryos injected with WeelB-S15D-phosphormimic mutants remained low and stable at $26-29 \mathrm{~h}$ post-hCG injection (Fig. 4C).

Microinjection of Wee1B-WT and Wee1B-S15A/D mutants overcomes $G_{2} / M$ transition in mouse embryos induced by $H$-89. In the presence of $40 \mu \mathrm{mol} / 1 \mathrm{H}-89$, the cleavage of mouse embryos was observed at 26-29 h post-hCG injection, and the cleavage rate was calculated at $30 \mathrm{~h}$ (Fig. 4D). The results demonstrated that the cleavage rate of embryos in controls induced by $\mathrm{H}-89$ increased markedly and embryos 
A
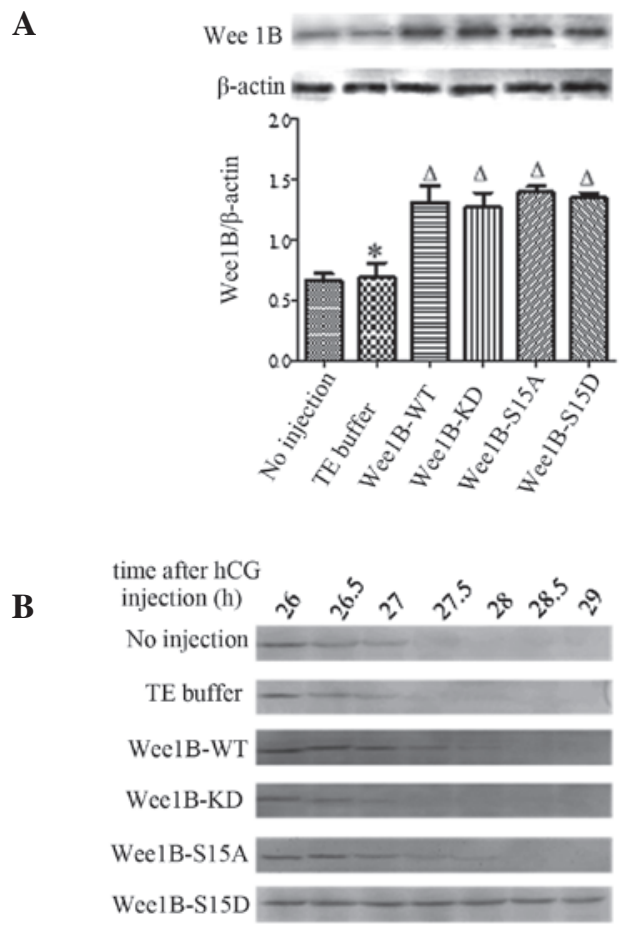

C
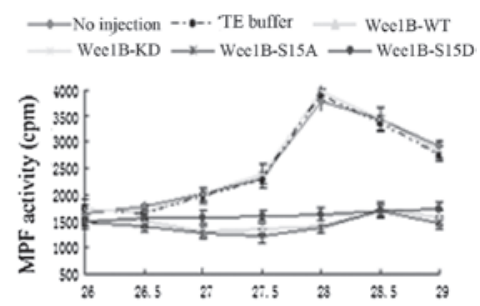

time after hCG injection (h)

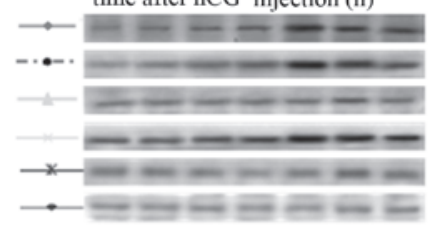

D

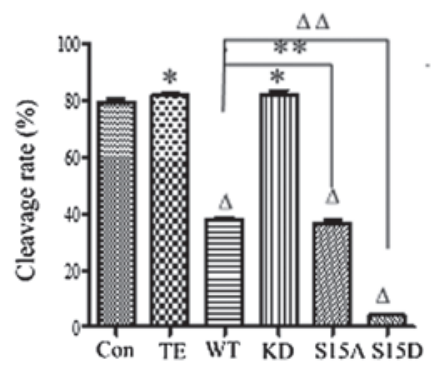

Figure 4. Wee1B-S15D blocks H-89 inhibition of PKA. (A) Western blotting analysis of WEE1B expression $5 \mathrm{~h}$ following microinjection of WeelB mRNA in the presence of $40 \mu \mathrm{mol} / 1 \mathrm{H}-89$. A total of $0.03 \mathrm{ng} \mathrm{mRNA}$ encoding WeelB-WT/KD and WeelB-S15A/S15D were microinjected into each embryo. Embryos in the control group were microinjected with or without TE buffer. Western blotting was performed using anti-WEE1B antibody in different microinjected groups. $\beta$-actin served as an internal control. Band intensities for WEE1B were quantified and normalized to $\beta$-actin level (bottom panel). Each value was expressed as mean \pm SEM from three independent experiments. ${ }^{\Delta} \mathrm{P}<0.01$ and ${ }^{\prime \prime} \mathrm{P}<0.05$, compared with the no injection group. (B) Western blotting analysis of the phosphorylation status of CDK1-Tyr 15 in the control and various WeelB mRNA-injected embryos in the presence of $40 \mu \mathrm{mol} / 1 \mathrm{H}-89$. The embryos were collected at 26, 26.5, 27, 27.5, 28, 28.5 and $29 \mathrm{~h}$ post-hCG injection. A total of 160 embryos were loaded onto each lane and fractionated on $12 \%$ SDS-PAGE. Gels were then transferred to the PVDF membrane and probed with CDK1-Tyr 15 antibody. Shown is a representative of three independent experiments. (C) MPF activity in embryos injected with various Wee1B mRNAs and controls. Embryos in the control group were microinjected with or without TE buffer. The embryos in the presence of $40 \mu \mathrm{mol} / 1 \mathrm{H}-89$ were collected at 26, 26.5, 27, 27.5, 28, 28.5 and $29 \mathrm{~h}$ post-hCG injection. For each point, 10 embryos were collected and MPF activity was examined by scintillation counting (upper panel) and autoradiography (bottom panel). Each value is expressed as the mean \pm SEM and shown is a representative of three independent experiments. (D) The cleavage rate in cultured mouse embryos injected with various WeelB mRNAs and controls at $30 \mathrm{~h}$ post-hCG in the presence of $40 \mu \mathrm{mol} / 1 \mathrm{H}-89$. The cleavage rate was calculated and each value was expressed as mean \pm SEM from three independent experiments. ${ }^{\Delta} \mathrm{P}<0.01$ and ${ }^{*} \mathrm{P}<0.05$, compared with the no injection group. WeelB-WT, WeelB-wild type; WeelB-KD, WeelB-kinase dead; MPF, $\mathrm{M}$ phase-promoting factor; $\mathrm{hCG}$, human chorionic gonadotropin; CDK1, cyclin-dependent kinase 1.

entered $\mathrm{M}$ phase at $27.5-28 \mathrm{~h}$ post-hCG injection, almost 79.2 (no injection) and $81.3 \%$ (TE injection) of embryos had developed into the two-cell stage at $30 \mathrm{~h}$ post-hCG injection. However, embryos injected with WeelB-WT and WeelB-S15A mRNAs resumed mitosis at $28.5 \mathrm{~h}$ post-hCG injection, and $\sim 40 \%$ of embryos had developed to the 2 -cell stage at $30 \mathrm{~h}$, markedly lower than that in the control $(\mathrm{P}<0.01)$. The embryos injected with WeelB-KD mutants entered M phase at $27.5-28 \mathrm{~h}$, and $82.13 \%$ of embryos had reached the two-cell stage at $30 \mathrm{~h}$ post-hCG injection, which was similar to the control; however, embryos injected with WeelB-S15D mRNA rarely entered into the two-cell stage at $30 \mathrm{~h}$ post-hCG injection. Taken together, these results demonstrate that Ser 15 phosphorylation of WEE1B is important in $\mathrm{G}_{2} / \mathrm{M}$ transition of mouse embryos.

\section{Discussion}

In the present study, we analyzed the regulation of mouse WEE1B and examined the role of Ser 15 of WEE1B in regulating the development of one-cell stage mouse embryos.

PKA is important in regulating the cell cycle progression of eukaryotic organisms. A study by Shimaoka et al suggested that continuous high PKA activity is a primary cause of the meiotic incompetence of pig-growing oocytes, and that this PKA activity is, not only caused by an insufficient expression level of PKA subunits, but may be attributed to more complex spatial-temporal regulation mechanisms (45). Our studies have demonstrated that PKA negatively regulates cell cycle progression in one-cell stage mouse embryos by inhibiting MPF (46) and CDC25B acts as a direct substrate of PKA (27). PKA activity oscillates with cell cycle progression. The activity of the free $\mathrm{C}$ subunit of PKA was high during interphase, decreased to a minimum level at the onset of mitosis and increased again at the metaphase-anaphase transition (46). PKA directly phosphorylates mouse WEE1B in a cAMP concentration-dependent manner, at least in vitro (21). Ser 15 in the $\mathrm{N}$ terminus of mouse WEE1B is a major PKA phosphorylation target, and phosphorylation at this site enhances the autophosphorylation activity of WEE1B and its ability to inhibit CDK1 and oocyte maturation (21). In the present study, to further examine the role of the Ser 15 site of WEE1B in regulating the development of one-cell stage mouse embryos, we demonstrated that overexpressed WeelB-WT and WeelB-S15A, not only inhibits the mitotic $\mathrm{G}_{2} / \mathrm{M}$ transition, but also decreases the cleavage rate of 
mouse embryos. However, inhibition by the S15A mutant did not differ from that of WeelB-WT. Since WeelB-KD is a kinase-dead mutant, Lys 237 of WeelB-WT was altered to Met, no significant difference with the control was observed. Overexpression of phosphor-mimic Wee1B-S15D mutants inhibited mitosis more efficiently than WeelB-WT and S15A mutant in the absence of dbcAMP and H-89, indicating that overexpression of WEE1B prevents the activation of MPF in the nucleus, which is a prerequisite for the induction of mitotic resumption. Residue Ser 15 of WEE1B is likely a direct target of PKA in mouse embryos.

To identify the PKA phosphorylation target of WEE1B, affinity-purified phosphor-specific and non-phosphor-specific antibodies were used to detect the phosphorylation status of WEE1B-Ser 15 in embryos at $G_{1}, S, G_{2}$ and $M$ phases by western blotting in the absence or presence of dbcAMP and/ or H-89. The results suggest that WEE1B-Ser 15 is phosphorylated at the $\mathrm{G}_{1}$ and $\mathrm{S}$ phases, whereas WEE1B-Ser 15 is dephosphorylated at the $\mathrm{G}_{2}$ and $\mathrm{M}$ phases in vivo. Taken together, our results indicate that PKA regulates the early development of mouse embryos by phosphorylation of Ser 15 whether dbcAMP is present or not. If PKA phosphorylated Ser 15 of WEE1B during the $\mathrm{G}_{1}$ and $\mathrm{S}$ phases, WEE1B kinase was activated to some extent, which is capable of inactivating MPF, resulting in the retardation of mitosis progression. By contrast, PKA levels are low in $\mathrm{G}_{2} / \mathrm{M}$ transition, dephosphorylation of Ser 15 in the $G_{2}$ phase by an unknown protein induced the inactivation of WEE1B, and MPF activity was activated by $\mathrm{CDC} 25 \mathrm{~B}$ phosphatase, thus resuming mitosis by the direct dephosphorylation of CDK1-Tyr 15 (27).

To directly test whether the inhibition of mouse embryos by Ser 15 of WEE1B was due to levels of endogenous PKA, specifically endogenous PKA activity was activated or inhibited and the role of WEE1B in one-cell stage mouse embryos was examined. The inhibitory effects of S15D mutant delays the re-entry of embryos into mitosis, which was strengthened compared with WeelB-WT and S15A mutant in the presence of dbcAMP and H-89. MPF activity remained at a relatively low level and the phosphorylation status of CDK1-Tyr 15 at indicated times in each group was coincident with the MPF activity. Therefore, the $15 \mathrm{D}$ mutant-enhanced inhibition of division rate of mouse embryos is due to direct phosphorylation of CDK1-Tyr 15 and the inhibition of MPF activity. Conversely, the cleavage rate of embryos induced by dbcAMP decreased markedly, and a low number of embryos reached the two-cell stage at $35 \mathrm{~h}$ post-hCG injection in the control group and WeelB-WT/KD and Wee1B-S15A mRNA-microinjected embryos, suggesting that PKA activation is responsible for the inhibition of MPF activity and enhanced CDK1-Tyr 15 phosphorylation status. By contrast, in the presence of $40 \mu \mathrm{mol} / 1$ $\mathrm{H}-89$, mouse embryos in the control group and those injected with WeelB-KD mRNA initiated mitosis rapidly, which suggests that PKA inhibition is responsible for the activation of MPF activity. However, embryos microinjected with WeelB-WT and WeelB-S15A/D mutants overcome $\mathrm{G}_{2} / \mathrm{M}$ transition induced by $\mathrm{H}-89$. Our results indicate that sustained activation or inhibition of PKA by treatment with dbcAMP or $\mathrm{H}-89$ into mouse embryos may prolong or promote interphase in control and WeelB-KD mRNA-injected embryos; however, overexpressed WeelB-WT and WeelB-S15A/D mutants lead to $\mathrm{G}_{2}$ arrest. The Wee1B-S15D mutant inhibited CDK1-Tyr 15 phosphorylation more potently than WeelB-WT and Wee1B-S15A. These results strongly suggest that PKA phosphorylates Ser 15 of WEE1B and this phosphorylation causes an inactivation of MPF activity by the direct phosphorylation of CDK1-Tyr 15 with a consequently enhanced inhibitory effect in embryo maturation.

Notably, WeelB-S15A did not exhibit a dominant-negative effect compared with the WeelB-WT in detecting MPF activity and cleavage rate in the absence or presence of dbcAMP and/ or H-89 in one-cell stage embryos, suggesting that endogenous WEE1B may be important in cell cycle progression. Therefore, the knockdown and overexpression of WeelB into mouse embryos concurrently may be a way to examine whether inhibition of mouse embryos occurs by phosphorylation of Ser 15 of WEE1B or due to the endogenous WEE1B. Further experiments are required to verify this hypothesis.

Taken together, the present study combined with our previous studies (27), provides new insights into the molecular mechanisms of $\mathrm{G}_{2} / \mathrm{M}$ transition involved in the early development of mammalian embryos. A decrease of cAMP in $\mathrm{G}_{2} / \mathrm{M}$ transition of one-cell stage embryos and inactivation of PKA (46) causes a rapid and progressive CDC25B translocation to the nucleus. Thus, the accumulation of $\mathrm{CDC} 25 \mathrm{~B}$ in the nucleus causes dephosphorylation and activation of the fraction of MPF that is shuttling into the nucleus (27). Following this, the activated MPF promotes the export of WEE1B to the cytoplasm by an unknown mechanism. The translocation of WEE1B functions as an amplification step to further promote the activation of the nuclear MPF until a threshold of activity sufficient for $\mathrm{G}_{2} / \mathrm{M}$ transition for WEE1B has no access to its MPF substrate and that this is sufficient to cause MPF activation. However, it is also possible that WEE1B becomes inactivated during the export process or during its accumulation in the cytosol (47), further suppressing its cytostatic activity for low PKA activity leading to the inactivation of WEE1B. CDC25B may then be transferred from the nucleus to the cytosol (27), WEE1B is also exported from the cytoplasm to the nucleus. The reciprocal translocation between CDC25B and WEE1B is the prerequisite for the first embryonic divisions.

\section{Acknowledgements}

The authors would like to thank Professor Marco Conti (University of California, San Francisco, USA) for providing mouse wild-type Wee1B and kinase-dead Wee1B and Dr Rongjian Su (Liaoning Medical University, Liaoning, China) for revising the manuscript and providing valuable advice and encouragement. This study was supported by the National Nature Science Foundation of China (81070489 and 81270654) and the Educational Commission of Liaoning Province of China (L2012303).

\section{References}

1. Nigg EA: Mitotic kinases as regulators of cell division and its checkpoints. Nat Rev Mol Cell Biol 2: 21-32, 2001.

2. Nurse P: Universal control mechanism regulating onset of M-phase. Nature 344: 503-508, 1990.

3. Coleman TR and Dunphy WG: Cdc2 regulatory factors. Curr Opin Cell Biol 6: 877-882, 1994. 
4. Lew DJ and Kornbluth S: Regulatory roles of cyclin dependent kinase phosphorylation in cell cycle control. Curr Opin Cell Biol 8: 795-804, 1996.

5. Nakajima $H$, Yonemura S, Murata $M$, Nakamura $N$, Piwnica-Worms $\mathrm{H}$ and Nishida E: Myt1 protein kinase is essential for Golgi and ER assembly during mitotic exit. J Cell Biol 181: 89-103, 2008.

6. Mueller PR, Coleman TR, Kumagai A and Dunphy WG: Myt1: a membrane-associated inhibitory kinase that phosphorylates Cdc2 on both threonine-14 and tyrosine-15. Science 270: 86-90, 1995.

7. Booher RN, Deshaies RJ and Kirschner MW: Properties of Saccharomyces cerevisiae weel and its differential regulation of p34CDC28 in response to G1 and G2 cyclins. EMBO J 12: 3417-3426, 1993

8. McGowan $\mathrm{CH}$ and Russell P: Human Weel kinase inhibits cell division by phosphorylating p34cdc2 exclusively on Tyr15. EMBO J 12: 75-85, 1993.

9. McGowan $\mathrm{CH}$ and Russell P: Cell cycle regulation of human WEE1. EMBO J 14: 2166-2175, 1995.

10. Mueller PR, Coleman TR and Dunphy WG: Cell cycle regulation of a Xenopus Weel-like kinase. Mol Biol Cell 6: 119-134, 1995.

11. Kornbluth S, Sebastian B, Hunter T and Newport J: Membrane localization of the kinase which phosphorylates p34cdc2 on threonine 14. Mol Biol Cell 5: 273-282, 1994.

12. Booher RN, Holman PS and Fattaey A: Human Myt1 is a cell cycle-regulated kinase that inhibits Cdc2 but not Cdk2 activity. J Biol Chem 272: 22300-22306, 1997.

13. Liu F, Stanton JJ, Wu Z and Piwnica-Worms H: The human Myt1 kinase preferentially phosphorylates $\mathrm{Cdc} 2$ on threonine 14 and localizes to the endoplasmic reticulum and Golgi complex. Mol Cell Biol 17: 571-583, 1997.

14. Liu F, Rothblum-Oviatt C, Ryan CE and Piwnica-Worms H: Overproduction of human Myt1 kinase induces a G2 cell cycle delay by interfering with the intracellular trafficking of Cdc2cyclin B1 complexes. Mol Cell Biol 19: 5113-5123, 1999.

15. Wells NJ, Watanabe N, Tokusumi T, Jiang W, Verdecia MA and Hunter T: The C-terminal domain of the $\mathrm{Cdc} 2$ inhibitory kinase Myt1 interacts with Cdc2 complexes and is required for inhibition of G(2)/M progression. J Cell Sci 112 (Pt 19): 3361-3371, 1999.

16. Hanna CB, Yao S, Patta MC, Jensen JT and Wu X: WEE2 is an oocyte-specific meiosis inhibitor in rhesus macaque monkeys. Biol Reprod 82: 1190-1197, 2010.

17. Shimaoka T, Nishimura T, Kano K and Naito K: Critical effect of pigWee1B on the regulation of meiotic resumption in porcine immature oocytes. Cell Cycle 8: 2375-2384, 2009.

18. Nakanishi $\mathrm{M}$, Ando $\mathrm{H}$, Watanabe $\mathrm{N}$, et al: Identification and characterization of human Wee1B, a new member of the Weel family of Cdk-inhibitory kinases. Genes Cells 5: 839-847, 2000.

19. Nishimura T, Shimaoka T, Kano K and Naito K: Insufficient amount of $\mathrm{Cdc} 2$ and continuous activation of Wee1 B are the cause of meiotic failure in porcine growing oocytes. J Reprod Dev 55: 553-557, 2009.

20. Han SJ and Conti M: New pathways from PKA to the Cdc2/cyclin B complex in oocytes: Wee1B as a potential PKA substrate. Cel Cycle 5: 227-231, 2006.

21. Han SJ, Chen R, Paronetto MP and Conti M: Wee1B is an oocyte-specific kinase involved in the control of meiotic arrest in the mouse. Curr Biol 15: 1670-1676, 2005.

22. Mueller PR and Leise WF 3rd: Measurement of Wee kinase activity. Methods Mol Biol 296: 299-328, 2005.

23. Islam MS, Kawase O, Hase S, Hoshi M and Matsumoto $M$ PKA activation in concert with ARIS and asterosap induces the acrosome reaction in starfish. Zygote 14: 329-340, 2006

24. Wang J and Liu XJ: Progesterone inhibits protein kinase A (PKA) in Xenopus oocytes: demonstration of endogenous PKA activities using an expressed substrate. J Cell Sci 117: 5107-5116, 2004

25. Pirino G, Wescott MP and Donovan PJ: Protein kinase A regulates resumption of meiosis by phosphorylation of $\mathrm{Cdc} 25 \mathrm{~B}$ in mammalian oocytes. Cell Cycle 8: 665-670, 2009.
26. Kovo M, Kandli-Cohen M, Ben-Haim M, Galiani D, Carr DW and Dekel N: An active protein kinase A (PKA) is involved in meiotic arrest of rat growing oocytes. Reproduction 132: 33-43, 2006.

27. Xiao J, Liu C, Hou J, et al: Ser149 is another potential PKA phosphorylation target of Cdc25B in $\mathrm{G} 2 / \mathrm{M}$ transition of fertilized mouse eggs. J Biol Chem 286: 10356-10366, 2011.

28. Schultz R: PKA and CDC25B: at last connected. Cell Cycle 8 : 516-517, 2009.

29. Zhang Y, Zhang Z, Xu XY, et al: Protein kinase A modulates Cdc25B activity during meiotic resumption of mouse oocytes. Dev Dyn 237: 3777-3786, 2008.

30. Okamoto K and Sagata N: Mechanism for inactivation of the mitotic inhibitory kinase Weel at M phase. Proc Natl Acad Sci USA 104: 3753-3758, 2007

31. Burrows AE, Sceurman BK, Kosinski ME, et al: The C.elegans Myt1 ortholog is required for the proper timing of oocyte maturation. Development 133: 697-709, 2006.

32. Maller JL, Butcher FR and Krebs EG: Early effect of progesterone on levels of cyclic adenosine $3^{\prime}: 5^{\prime}$-monophosphate in Xenopus oocytes. J Biol Chem 254: 579-582, 1979.

33. Conti M, Andersen CB, Richard FJ, Shitsukawa K and Tsafriri A: Role of cyclic nucleotide phosphodiesterases in resumption of meiosis. Mol Cell Endocrinol 145: 9-14, 1998.

34. Conti M, Andersen CB, Richard F, et al: Role of cyclic nucleotide signaling in oocyte maturation. Mol Cell Endocrinol 187: $153-159,2002$.

35. Dekel N: Protein phosphorylation/dephosphorylation in the meiotic cell cycle of mammalian oocytes. Rev Reprod 1: 82-88, 1996.

36. Okamoto K, Nakajo N and Sagata N: The existence of two distinct Weel isoforms in Xenopus: implications for the developmental regulation of the cell cycle. EMBO J 21: 2472-2484, 2002

37. Parker LL and Piwnica-Worms H: Inactivation of the p34cdc2cyclin B complex by the human WEE1 tyrosine kinase. Science 257: 1955-1957, 1992 .

38. Hogan B, Beddington R, Constantini F and Lacy E: Manipulating the mouse embryo: a laboratory manual. Cold Harbor Laboratory Press, New York, 1986.

39. Zhang Z, Su WH, Feng C, et al: Polo-like kinase 1 may regulate G2/M transition of mouse fertilized eggs by means of inhibiting the phosphorylation of Tyr 15 of Cdc2. Mol Reprod Dev 74: $1247-1254,2007$

40. Cui C, Zhao H, Zhang Z, et al: $\mathrm{CDC} 25 \mathrm{~B}$ acts as a potential target of PRKACA in fertilized mouse eggs. Biol Reprod 79: 991-998, 2008.

41. Gallicano GI, McGaughey RW and Capco DG: Activation of protein kinase $\mathrm{C}$ after fertilization is required for remodeling the mouse egg into the zygote. Mol Reprod Dev 46: 587-601, 1997.

42. Xu XY, Zhang Z, Su WH, et al: Involvement of the p110 alpha isoform of PI3K in early development of mouse embryos. Mol Reprod Dev 76: 389-398, 2009.

43. Vitolo OV, Sant'Angelo A, Costanzo V, Battaglia F, Arancio O and Shelanski M: Amyloid $\beta$-peptide inhibition of the PKA/CREB pathway and long-term potentiation: reversibility by drugs that enhance cAMP signaling. Proc Natl Acad Sci USA 99: 13217$13221,2002$.

44. Yamada T, Matsuda K and Uchiyama M: Atrial natriuretic peptide and cGMP activate sodium transport through PKA-dependent pathway in the urinary bladder of the Japanese tree frog. J Comp Physiol B 176: 203-212, 2006.

45. Shimaoka T, Nishimura T, Kano K and Naito K: Analyses of the regulatory mechanism of porcine WEE1B: the phosphorylation sites of porcine WEE1B and mouse WEE1B are different. J Reprod Dev 57: 223-228, 2010

46. Yu B, Wang Y, Liu Y, et al: Protein kinase A regulates cell cycle progression of mouse fertilized eggs by means of MPF. Dev Dyn 232: 98-105, 2005

47. Oh JS, Han SJ and Conti M: Wee1B, Myt1, and Cdc25 function in distinct compartments of the mouse oocyte to control meiotic resumption. J Cell Biol 188: 199-207, 2010. 